\title{
Targeting fibroblast activation protein inhibits tumor stromagenesis and growth in mice
}

\author{
Angélica M. Santos, ${ }^{1}$ Jason Jung, ${ }^{2}$ Nazneen Aziz, ${ }^{3}$ Joseph L. Kissil,, ${ }^{1}$ and Ellen Puré1,4 \\ ${ }^{1}$ The Wistar Institute, Philadelphia, Pennsylvania, USA. ${ }^{2}$ Cell and Molecular Biology Graduate Group, University of Pennsylvania, Philadelphia, \\ Pennsylvania, USA. ${ }^{3}$ Point Therapeutics Inc., Boston, Massachusetts, USA. ${ }^{4}$ Ludwig Institute for Cancer Research, Philadelphia, Pennsylvania, USA.
}

\begin{abstract}
Membrane-bound proteases have recently emerged as critical mediators of tumorigenesis, angiogenesis, and metastasis. However, the mechanisms by which they regulate these processes remain unknown. As the cell surface serine protease fibroblast activation protein (FAP) is selectively expressed on tumor-associated fibroblasts and pericytes in epithelial tumors, we set out to investigate the role of FAP in mouse models of epithelialderived solid tumors. In this study, we demonstrate that genetic deletion and pharmacologic inhibition of FAP inhibited tumor growth in both an endogenous mouse model of lung cancer driven by the K-ras ${ }^{\mathrm{G} 12 \mathrm{D}}$ mutant and a mouse model of colon cancer, in which CT26 mouse colon cancer cells were transplanted into immune competent syngeneic mice. Interestingly, growth of only the $\mathrm{K}$-ras ${ }^{\mathrm{G} 12 \mathrm{D}}$-driven lung tumors was also attenuated by inhibition of the closely related protease dipeptidyl peptidase IV (DPPIV). Our results indicate that FAP depletion inhibits tumor cell proliferation indirectly, increases accumulation of collagen, decreases myofibroblast content, and decreases blood vessel density in tumors. These data provide proof of principle that targeting stromal cell-mediated modifications of the tumor microenvironment may be an effective approach to treating epithelial-derived solid tumors.
\end{abstract}

\section{Introduction}

Tumors are composed of heterogeneous populations of cells, including transformed cells and a multitude of untransformed cells. Although the prevalence of different cell types varies among tumors and at different stages of tumor progression, they include infiltrating inflammatory and immune cells, endothelial cells and mesenchymal-derived smooth muscle cells, pericytes, and tumor-associated fibroblasts (TAFs), which are referred to herein collectively as stromal cells. TAFs are a heterogeneous population that can be phenotypically distinguished from normal fibroblasts. Fibroblast activation protein (FAP) has emerged as a marker of reactive fibroblasts in tumors as well as granulation tissue and in fibrotic lesions. Although the phenotypic and functional heterogeneity among TAFs is yet to be fully explored, at least a subset of TAFs have been characterized as myofibroblasts, based on expression of $\alpha$ SMA.

Stromal cells communicate among themselves as well as with cancer cells and inflammatory and immune cells directly through cell contact and indirectly through paracrine/exocrine signaling, proteases, and modulation of the ECM. This complex communications network is pivotal to providing the appropriate microenvironment to support tumorigenesis, angiogenesis, and metastasis $(1,2)$. Considering the key role of the microenvironment in tumor development, identification of stromal targets for cancer therapeutics is of great interest and could provide strategies that will complement therapies directed against cancer cells. Among these potential targets is an array of proteases (3).

Proteases are important factors in the pathophysiology of tumors, having requisite roles in angiogenesis and metastasis. The major classes of endopeptidases involved in ECM degradation

Authorship note: Joseph L. Kissil and Ellen Puré contributed equally to this work. Conflict of interest: Ellen Puré received research support in 2007 from Point Therapeutics Inc

Citation for this article: J. Clin. Invest. 119:3613-3625 (2009). doi:10.1172/JCI38988. include serine (such as the plasminogen activator, uPA), cysteine (cathepsins), aspartyl, and MMPs $(4,5)$. Numerous reports have demonstrated increased expression of ECM degrading enzymes, including type IV collagenase (MMP-2), cathepsin B, cathepsin D, and serine proteases, such as uPA in tumor cells (6), but the benefit of targeting the activity of proteases in cancer has yet to be established. Also, the widespread expression of many of these enzymes is likely to limit their potential as therapeutic targets. In contrast, FAP (also called FAP $\alpha$ or seprase) has recently gained attention as a potential target, due to its tightly regulated expression in the tumor stroma and structurally defined proteolytic activity (7-11); however, its function in tumors is largely unknown.

FAP is a type II transmembrane cell surface protein belonging to the post-proline dipeptidyl aminopeptidase family, sharing the highest similarity with dipeptidyl peptidase IV (DPPIV/CD26). FAP is expressed selectively by TAFs and pericytes in more than $90 \%$ of human epithelial cancers examined (12-16). It is also expressed during embryonic development (17), in tissues of healing wounds (18), and in chronic inflammatory and fibrotic conditions such as liver cirrhosis $(19,20)$ and idiopathic pulmonary fibrosis (21), as well as on bone and soft tissue sarcomas $(16,22)$ and some melanoma (23). Expression of FAP is not however detected in benign lesions or normal adult tissues $(23,24)$, while DPPIV is more widely expressed in a variety of cell types (reviewed in refs. $25,26)$. In vitro studies have shown that FAP has both dipeptidyl peptidase $(19,24)$ and endopeptidase activity $(8,10,27)$, including a collagenolytic activity capable of degrading gelatin $(28,29)$ and type I collagen $(27,30)$, but its in vivo substrate(s) is yet to be defined. Based on the highly regulated expression and restricted distribution of FAP, it has been suggested that FAP inhibition may be useful in cancer therapeutics.

Although some experimental evidence suggesting FAP promotes tumorigenesis has been reported, the models used in these studies largely failed to recapitulate the potentially important interactions between mesenchymal-derived stromal cells, 
A
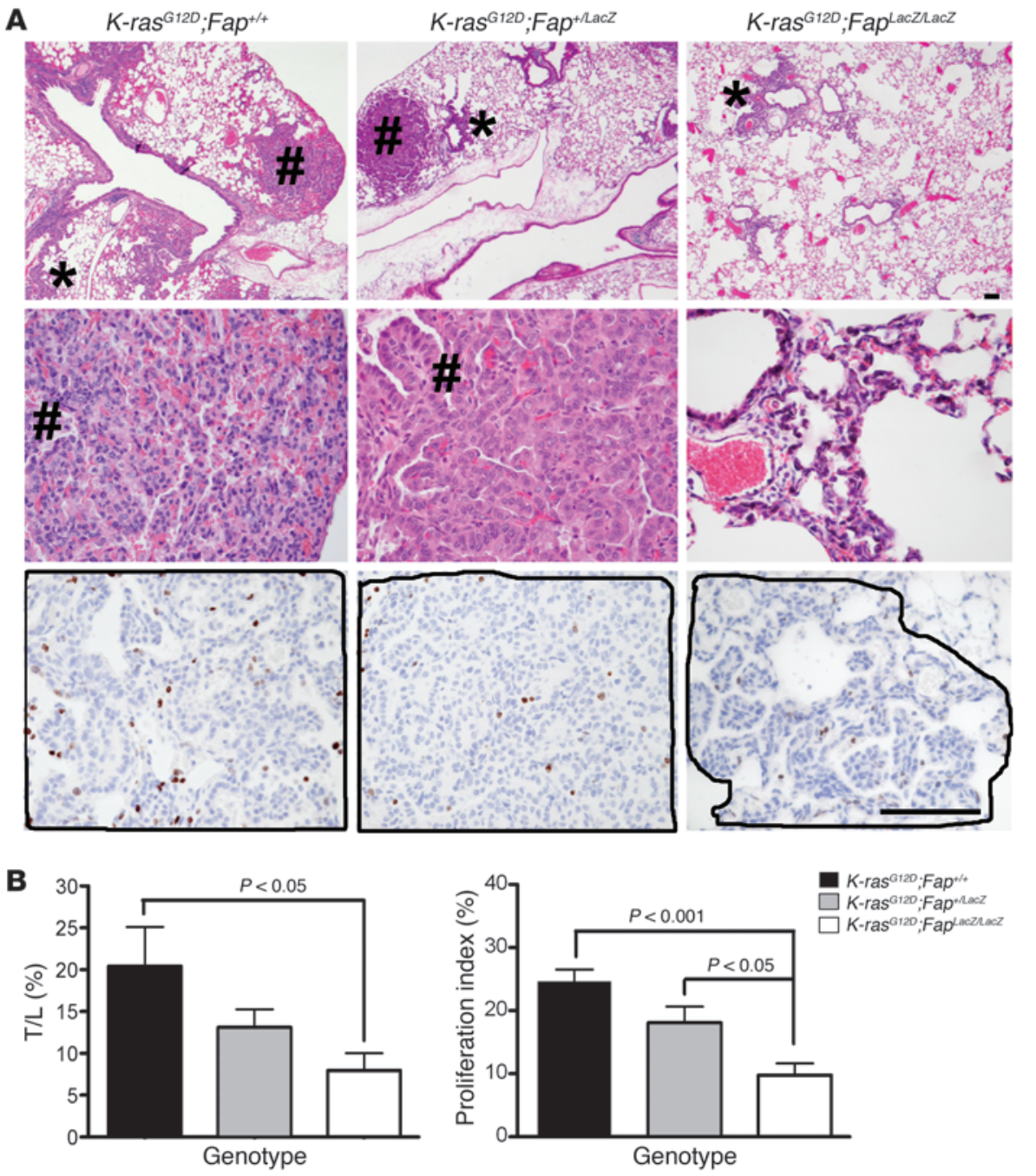

\section{Figure 1}

Development of lung tumors in $L S L-K$ ras $^{\mathrm{G} 12 D ;} ; \mathrm{Fap}^{+/+}$, LSL-K-ras G12D;Fap ${ }^{+/ L a c Z}$, and LSL-K-ras ${ }^{G 12 D ; F a p}{ }^{L a c Z / L a c Z}$ mice. (A) Representative sections from each genotype at 8 weeks after Ad-Cre infection. Regions of hyperplasia (asterisks) and adenomas (pound symbols) are indicated. Original magnification, $\times 4$ (top row); $\times 40$ (bottom 2 rows). Scale bar: $100 \mu \mathrm{m}$. Images that display areas of Ki67 staining, shown in the bottom row of panels, were selected based on similarity of tumor content (indicated by solid lines) between genotypes, whereas H\&E-stained sections show randomly selected representative areas, unrelated to those shown for Ki67. (B) Tumor-to-lung area (T/L) ratio in $L S L-K$-ras ${ }^{G 12 D} ;$ Fap $^{+/+}$, LSL-K-ras ${ }^{G 12 D} ; F^{\prime}{ }^{+/ L a c Z}$, and $L S L-K$-ras ${ }^{G 12 D}$; Fap ${ }^{L a c Z / L a c Z}$ mice at 8 weeks after Ad-Cre infection $(n=11)$ and proliferative index, which is calculated as percentage of Ki67positive cells in the indicated number of animals for each genotype ( $n=5$ animals). Results are expressed as mean \pm SEM. tumor cells and immune and inflammatory cells that typically occur in tumors. For example, a number of these studies used xenografts of human tumor cells ectopically overexpressing FAP, or an enzymatically inactive mutant, transplanted into immune incompetent mice $(31,32)$ despite the fact that in primary tumors, FAP is expressed only by the TAFs and pericytes and not by tumor cells. Furthermore, the mechanisms involved were not defined. To determine whether FAP promotes tumorigenesis and understand the molecular mechanism by which this might occur under more relevant pathophysiologic conditions, we studied the impact of genetic deletion of FAP and pharmacologic inhibition of its enzymatic activity in both syngeneic transplant and endogenous mouse tumor models (33-37). We herein demonstrate that endogenous FAP expressed on tumor stromal cells promotes tumor progression via its enzymatic activity. Our results indicate that FAP is a major source or regulator of collagenase activity in tumors, FAP promotes tumor growth by indirectly promoting tumor cell proliferation, and that FAP is required for tumor stromagenesis and vascularization. In addition to providing insight into the mechanisms by which FAP promotes tumorigenesis, these results suggest that inhibition of FAP enzymatic activity warrants further investigation as a potential therapeutic approach.

\section{Results}

Development of endogenous lung tumors is inbibited in FAP-deficient

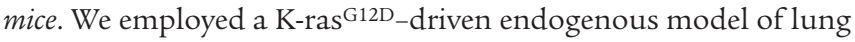
adenocarcinoma to examine the consequences of the loss of FAP on tumorigenesis. In this model, lung tumorigenesis is driven by the conditional activation of an oncogenic allele of $K-$ ras $^{G 12 D}$. Activation of the $K$-ras ${ }^{G 12 D}$ allele is achieved by delivery of adenovirus expressing the Cre recombinase (Ad-Cre), resulting in recombination and removal of a transcriptional STOP element in the Lox-Stop-Lox (LSL) cassette (34). Fap-null mice, generated by LacZ knockin, are viable (17), with normal lung histology (Supplemental Figure 1A; supplemental material available online with this article; doi:10.1172/JCI38988DS1) and similar basal collagen content to littermate $\mathrm{Fap}^{+/+}$control mice (Supplemental Figure 1B). The overall morphology of lungs from $L S L-K$-ras ${ }^{G 12 D} ; \mathrm{Fap}^{+/+}$, $L S L-K$-ras ${ }^{G 12 D} ; F_{a} p^{+/ L a c Z}$, and $L S L-K$-ras ${ }^{G 12 D} ; F_{a} p^{L a c Z / L a c Z}$ littermates in the absence of Ad-Cre was similar (data not shown). Ad-Cre induced tumors in all genotypes, but reduced tumor burden in the absence of FAP was evident upon macroscopic inspection of lungs (Supplemental Figure 1C). By 8 weeks after Ad-Cre instillation $\left(2.5 \times 10^{7} \mathrm{PFU} / \mathrm{mouse}\right)$, extensive epithelial hyperplasia of the alveolar region (11 of 11 mice), adenomas (11 of 11 mice), and incidental pneumocyte hyperplasia ( 2 of 11 mice) were already evident in 


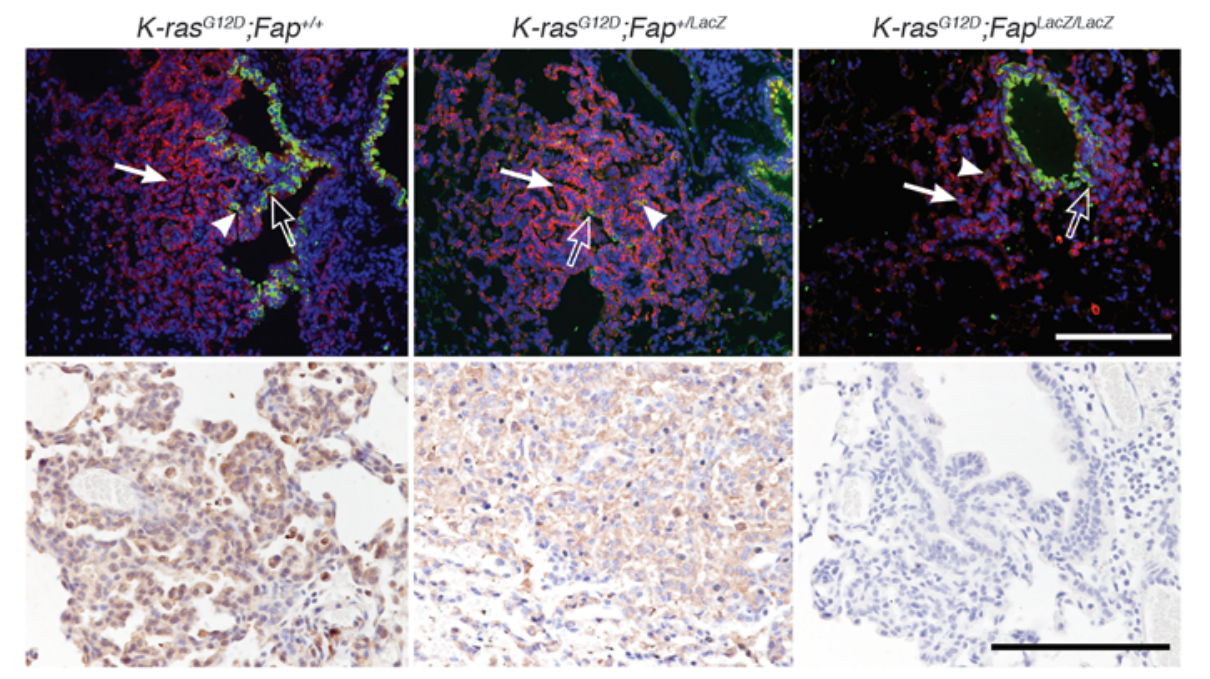

\section{Figure 2}

Immunophenotype of tumors in LSL-K-ras ${ }^{G 12 D}$; Fap mice. Immunofluorescence of CC10 (Clara cells, green; examples indicated by open arrows), SP-C (alveolar type II cells, red; examples indicated by filled arrows), and CC10/SP-C double-positive cells (yellow; examples indicated by arrowheads) (top row). FAP immunostaining of K-ras G12D-driven lung tumors (bottom row). Original magnification, $\times 20$ (top row); $\times 40$ (bottom row). Scale bars: $100 \mu \mathrm{m}$.
$L S L-K$-ras ${ }^{G 12 D} ; \mathrm{Fap}^{+/+}$mice, consistent with previous reports $(34,36)$. In contrast, $L S L-K$-ras ${ }^{G 12 D} ; F p^{+/ L a c Z}$ mice exhibited localized hyperplasia (10 of 10 mice) and some adenomas (6 of 10 mice), while $L S L-K$ ras $^{G 12 D} ; F^{2} p^{L a c Z / L a c Z}$ showed only small lesions of focal hyperplasia with incidental adenomas identified in only 4 of 11 mice in this group (Figure 1A). Overall, the tumor/lung volume ratio was significantly decreased from $20.4 \%$ in $L S L-K$-ras ${ }^{G 12 D} ; \mathrm{Fap}^{+/+}$mice to $7.9 \%(P=0.02)$ in $L S L-K$-ras ${ }^{G 12 D} ; F_{a} P^{L a c Z / L a c Z}$ mice (Figure 1B, left). Although, tumor burden in FAP heterozygous mice was not statistically different compared to $\mathrm{Fap}^{+/+}$mice, there was a trend toward reduced tumorigenesis (Figure 1B, left). Analysis of mortality rates indicated a significant delay in tumor-associated mortality in LSL-K-ras ${ }^{G 12 D}$;Fap ${ }^{L a c Z / L a c Z}$ compared with $L S L-K$-ras ${ }^{G 12 D} ; F^{2} p^{+/+}$mice. Specifically, median survival increased from 195 days for $L S L-K$ $\mathrm{ras}^{\mathrm{G} 12 \mathrm{D}} ; \mathrm{Fap}^{+/+}$mice to 233 days and 333 days for $L S L-K$-ras ${ }^{\mathrm{G} 12 \mathrm{D}} ; \mathrm{Fap} \mathrm{p}^{+/ L a c Z}$ and $L S L-K$-ras ${ }^{G 12 D} ; F_{a p}{ }^{L a c Z / L a c Z}$ mice, respectively. As shown in Supplemental Figure 1D, 5 of $11(\sim 45 \%) L S L-K-$ ras $^{G 12 D} ; \mathrm{Fap}^{+/+}$mice succumbed by 191 days after Ad-Cre instillation compared with 3 of $16(\sim 19 \%)$ of $L S L-K-$ ras $^{G 12 D} ; F^{2} p^{L a c Z / L a c Z}$ mice. Furthermore, all $L S L-K$-ras ${ }^{G 12 D} ; \mathrm{Fap}^{+/+}$mice succumbed by day 249, while 10 of 16 $L S L-K$-ras ${ }^{G 12 D} ; F^{2} p^{L a c Z / L a c Z}$ mice (63\%) were still alive at day 300 (Supplemental Figure 1D), although all eventually succumbed by day 410. Importantly, the reduced tumor growth was associated with a reduction in the proliferative index of tumors based on staining with Ki67 (Figure 1A, bottom, and Figure 1C, right), while the frequencies of apoptotic cells observed were comparable in tumors in the control and FAP-deficient mice (data not shown).

To compare the phenotype of the tumor cells in $L S L-K$-ras ${ }^{G 12 D}$; $\mathrm{Fap}^{+/+}, L S L-K$ ras $^{\mathrm{G} 12 D} ; \mathrm{Fap}^{+/ L a c Z}$, and $L S L-K$ ras $^{G 12 D} ; \mathrm{Fap}^{\mathrm{LacZ} / L a c Z}$ mice, sections were double stained for Clara cell-specific protein (CC10) and type II pneumocyte surfactant protein-C (SP-C). CC10 and SP-C are specific markers for bronchoalveolar epithelium, respectively (38). We found that the tumors in mice of all 3 genotypes were composed of SP-C $\mathrm{C}^{+}, \mathrm{CC} 0^{+}$, and $\mathrm{CC} 10^{+} \mathrm{SP}_{-} \mathrm{C}^{+}$tumor cells (Figure 2, top). Moreover, expression of FAP (by immunohistochemistry; Figure 2, bottom) and LacZ (by histologic detection of $\beta$-galactosidase activity; data not shown) demonstrated that TAFs (based on transcriptional activity of the FAP loci) were present in tumors at all stages. We did not detect expression of FAP in normal lung tissue by immunohistochemistry (data not shown). Thus, although, deletion of FAP resulted in reduced tumor burden, as evidenced by the reduced tumor/lung volume ratio, it did not appear to impact significantly the phenotype of the tumor cells.

The growth of syngeneic transplanted CT26 tumors is reduced in FAPdeficient mice. Inhibition of tumor growth of the endogenous tumors in Fap-null mice may be due to reduced tumor initiation or tumor progression. Therefore, to investigate whether deletion of FAP could inhibit tumor progression after tumor initiation and to test whether FAP promotes the growth of other tumor types, we extended our studies to a syngeneic transplanted mouse tumor model. Importantly, we did not detect Fap mRNA or protein in cultured CT26 cells (Supplemental Figure 2A and data not shown). In contrast, significant levels of Fap mRNA (Supplemental Figure 2A) and protein (Supplemental Figure 2B) were detected in the CT26 transplanted tumors. Consistent with previous reports in xenograft models as well as primary human epithelial carcinomas such as colon $(15,39,40)$, breast (16), and pancreas (12), these data, taken together with the pattern of FAP expression in tumor sections (Supplemental Figure 2B, left), indicated that endogenous FAP is induced specifically on host-derived TAFs and pericytes but not expressed in the transplanted tumor cells themselves. We then compared the growth of CT26 colon cancer cells injected s.c. into immune competent $\mathrm{Fap}^{+/+}$and Fap $\mathrm{LacZ}^{\mathrm{LacZ}}$ BALB/c mice. CT26 tumor growth was markedly reduced in Fapnull mice (Figure 3A), similar to our results in the endogenous lung tumor model. As shown above in the endogenous model, the decrease in CT26 tumor growth in FAP-deficient mice was associated with a decrease in tumor cell proliferation (Figure 3, $\mathrm{B}$ and $\mathrm{C}$ ), while the incidence of apoptotic cells was not affected (Supplemental Figure 3, A and C).

Pharmacologic inhibition of FAP protease activity inbibits tumorigenesis. We next investigated the impact of inhibiting FAP enzymatic activity in both the endogenous lung tumor model and transplanted CT26 tumors using a pharmacologic approach. $L S L-$ K-ras ${ }^{\mathrm{G} 12 \mathrm{D}} ; \mathrm{Fap}^{+/+}$mice were administered Ad-Cre as described above. Four weeks after infection with Ad-Cre, mice were randomly sorted into 3 groups and treated with PT630 (GluBoroPro dipeptide, known to inhibit FAP and the closely related DPPIV; ref. 41; patent application publication no. US 2007/0072830 A; Supplemental Figure 2D), LAF237 (Vildagliptin, a DPPIV inhibitor; refs. 42, 43) or saline (vehicle control) for an additional 4 weeks. PT630 inhibits FAP and DPPIV with $K_{i}$ in the nanomolar range (FAP with 
A
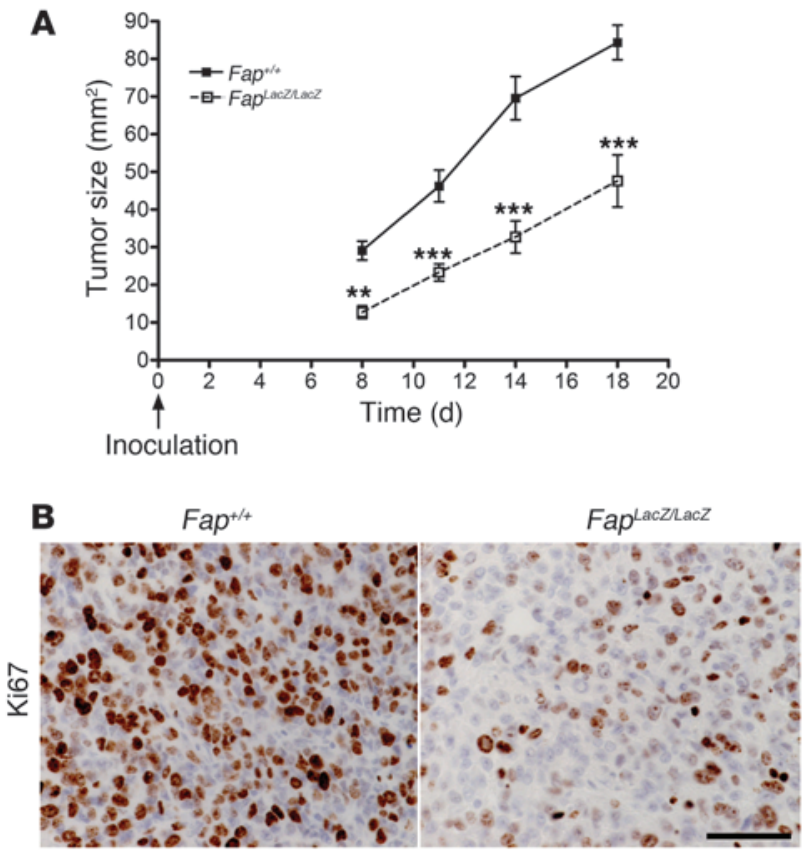

C

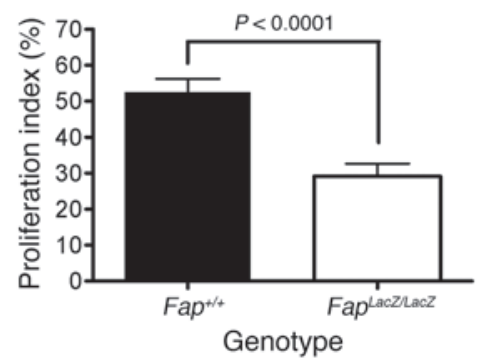

$\mathrm{IC}_{50}$ of $23 \mathrm{nM}$ and $K_{i}$ of $5 \mathrm{nM}$ and DPPIV with an $\mathrm{IC}_{50}$ of $3 \mathrm{nM}$; ref. 41), while LAF237 (Vildagliptin) is a potent and selective competitive inhibitor of DPPIV $\left(K_{m}, 1.4 \times 10^{5} \mathrm{M}^{-1} \mathrm{~s}^{-1} ; K_{i}, 17 \mathrm{nM} ; \mathrm{IC}_{50}, 4-8\right.$ nM; refs. 42, 43). LAF237 inhibits DPPIV at micromolar concentrations for DPPIV but does not inhibit FAP, DPPII, prolyl oligopeptidase, or aminopeptidase (42). Therefore, LAF237 was used to discriminate between the effects of FAP and DPPIV. Interestingly, LAF237 and PT630 both efficiently reduced the lung tumor burden compared with the vehicle treated control group (Figure 4, A and B). Although in another independent experiment, tumor burden was again reduced in $L S L-K$-ras ${ }^{G 12 D} ; F^{2} P^{L a c Z / L a c Z}$ mice compared with $L S L-K$ ras $^{G 12 D} ; \mathrm{Fap}^{+/+}$mice $(P<0.001)$ as expected, PT630 treatment of the $L S L-K$-ras ${ }^{G 12 D} ; F a p^{L a c Z / L a c Z}$ mice had no impact on tumor burden when compared with $L S L-K$-ras ${ }^{G 12 D} ; F a p^{L a c Z / L a c Z}$ mice treated with vehicle control, demonstrating that the effects of PT630 on tumor growth were FAP dependent (Supplemental Figure 4).

Although the genetic data indicated that deletion of FAP was sufficient to attenuate tumor growth, the results from these pharmacologic studies indicate that selective inhibition of DPPIV is also sufficient to inhibit tumor growth in this model. Therefore, we investigated the expression profile of DPPIV in the lungs prior to and after administration of Ad-Cre. Interestingly, immunohistochemical analysis established that DPPIV is indeed expressed in control lungs, both lungs from wild-type mice treated with Ad-Cre and uninfected $L S L-K-r a s^{G 12 D}$ mice (data not shown) as well as in lungs from mice bearing $\mathrm{K}$-ras ${ }^{\mathrm{G} 12 \mathrm{D}}$-driven tumors

\section{Figure 3}

CT26 tumor growth and tumor cell proliferation are inhibited in FAPdeficient mice. (A) CT26 tumor cells were injected s.c. in $\mathrm{Fap}^{+/+}$and FAP-null BALB/c mice. Tumor size was measured using calipers ( $n=14-15$ animals per genotype in 2 independent experiments). ${ }^{* *} P<0.001$; ${ }^{* *} P<0.0001$. (B) Ki67 immunohistochemistry and (C) proliferative index of CT26 tumors grown in $\mathrm{Fap}^{+/+}$and Fap LacZ/LacZ mice ( $n=5$ animals per genotype). Original magnification, $\times 60$. Scale bar: $50 \mu \mathrm{m} . P<0.0001$. Results are expressed as mean \pm SEM.

(Supplemental Figure 5); importantly, in the latter case the staining was associated with nontumor cells. Thus, in contrast to FAP, DPPIV is constitutively expressed in lung, rather than induced in response to tumor, but can nonetheless promote tumor growth.

We also determined the effect of PT630 and LAF237 on the growth of CT26 tumors in BALB/c mice. Two days after CT26 tumor cells were injected s.c., the mice were randomly divided into 3 groups and treated with saline, PT630, or LAF237. PT630, but not LAF237, significantly reduced CT26 tumor growth compared with vehicle control $(P<0.0008$ and $P<0.0001$ at days 14 and 17 , respectively; Figure 4C and Supplemental Figure 6). A potent antitumor effect of PT630 was indicated by treatment/control growth ratios of less than 0.40 , i.e., 0.37 and 0.27 on days 14 and 17 , respectively, which correspond to reductions in tumor size of $63 \%$ and $73 \%$. No apparent toxicity was observed in any of the animals treated at the indicated doses of the inhibitors. As was the case in the endogenous lung tumor model, PT630 had no effect on

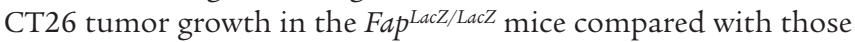
treated with vehicle control alone (Supplemental Figure 6). Taken together these data indicate that FAP activity, but not DPPIV activity, promotes the growth of CT26 tumors and that the effects of PT630 are indeed mediated by FAP in this tumor model as well.

To determine whether the effect of PT630 on tumor growth was associated with inhibition of FAP enzymatic activity, we compared the tumor-associated FAP activity between the vehicle control- and drug-treated groups. CT26 tumor extracts from PT630-, LAF237-, and vehicle control-treated mice were harvested at different times in order to analyze tumors of similar size (days 10-12, days $11-14$, and days $16-20$, respectively, corresponding to tumor sizes of between 40 and $50 \mathrm{~mm}^{2}$ ) and tested for enzymatic activity. FAP activity, determined using Z-Gly-Pro-AMC as substrate, in tumors isolated from animals that were treated with PT630 was decreased 54\% compared with those from vehicle control-treated mice $(P<0.05$; Figure $4 \mathrm{D})$. The level of FAP activity associated with tumors from animals treated with LAF237 on the other hand was not significantly different from that of the vehicle control group. The decrease in protease activity was due to PT630 reducing FAP activity, as opposed to FAP expression at either the protein (Figure 4E) or mRNA levels (Supplemental Figure 2C and Supplemental Methods), which was comparable in extracts of tumors from all 3 groups of mice. Importantly, these data indicate that the tumorpromoting activity of FAP is mediated via its enzymatic activity and that even incomplete inhibition of FAP enzymatic activity is sufficient to impact tumor growth.

Similar to the effect of genetic deletion of FAP, PT630 treatment in both tumor models and LAF237 treatment in the endogenous lung tumor model inhibited proliferation (Figure 5). The proliferation indices of the CT26 tumors were determined based on Ki67 staining of sections of tumors from the 3 groups of CT2 6 tumor-bearing mice harvested at different time points (vehicle control, days 10-12; 


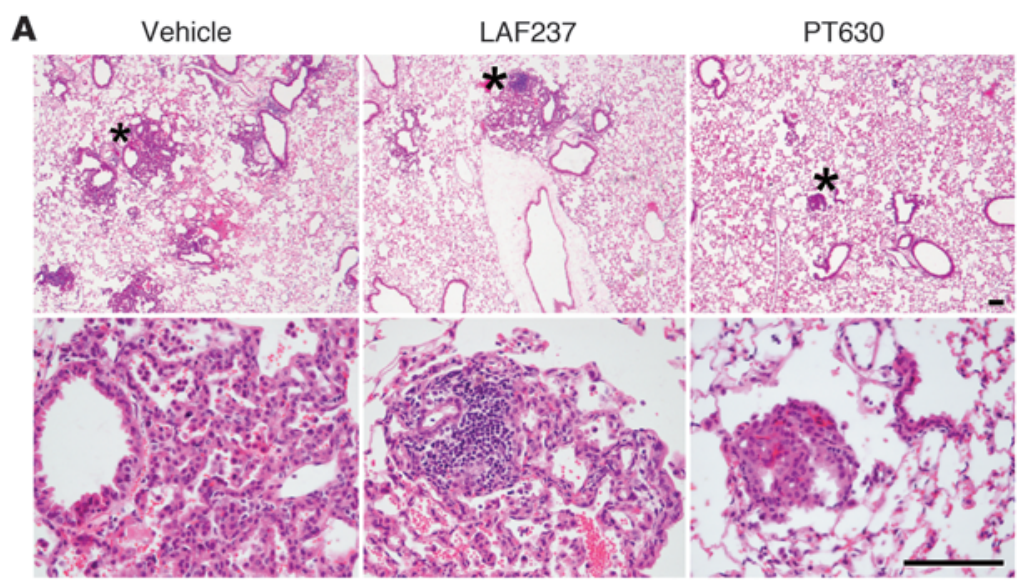

B

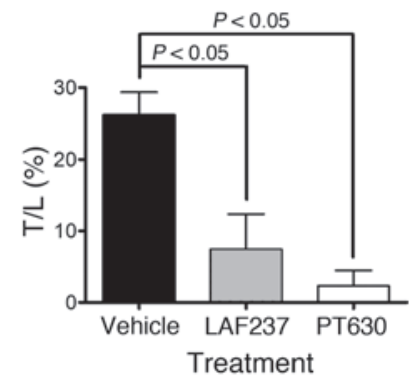

D

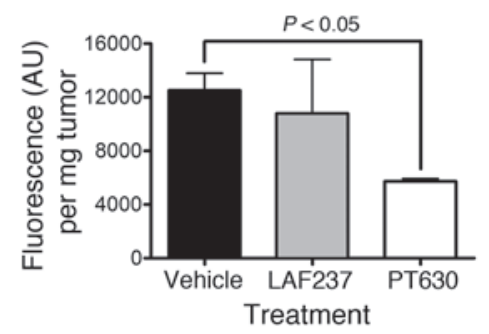

C

E

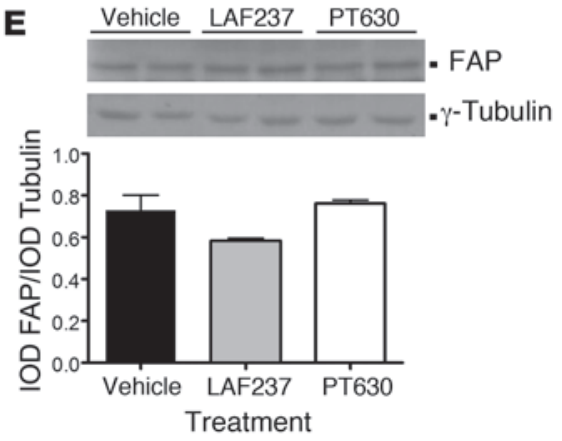

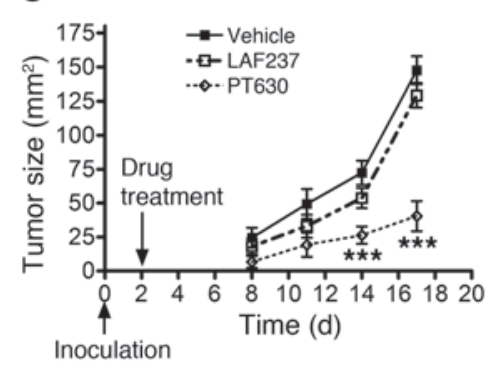

\section{Figure 4}

Effect of inhibition of FAP and/or DPPIV on tumor growth. (A) Representative H\&E-stained sections of lung from $L S L-K$-ras ${ }^{G 12 D}$ mice treated with vehicle, LAF237, or PT630 (top row). Higher-magnification views of regions indicated by asterisks are shown below (bottom row). Original magnification, $\times 4$ (top row); $\times 40$ (bottom row). Scale bar: $100 \mu \mathrm{m}$. (B) Treatment with PT630 and LAF237 reduced formation of K-ras ${ }^{\mathrm{G} 12 \mathrm{D}}$ driven lung tumors ( $n=5$ animals per group). Results are expressed as mean \pm SEM. (C) PT630 treatment inhibited CT26 tumor growth. Mice were treated by oral gavage with vehicle control, LAF237, or PT630 twice daily, starting on day 2, after tumor cell inoculation. Data represent mean $\pm \operatorname{SEM}(n=11$ animals per group in 2 independent experiments). ${ }^{\star \star \star} P<0.0001$ versus vehicle control. (D and $\mathbf{E}$ ) Treatment of mice with PT630 inhibited tumor-associated FAP enzymatic activity measured ex vivo (D), but not protein levels (E), as shown by immunoblotting (top panel) and corresponding densitometry (bottom panel). Results are expressed as mean \pm SEM $(n=10)$. IOD, image optical density.
LAF237, days 11-14; and PT630, days 16-20) in order to analyze tumors of approximately the same size (Figure 5, A and C). Proliferation was significantly reduced in tumor sections from animals treated with PT630 $(P<0.05)$ compared with tumors from animals treated with vehicle control or LAF237 ( $P<0.01$; Figure $5 C)$. The incidence of apoptosis as assessed by active caspase- 3 immunostaining was quite variable but did not differ significantly in either the tumors from PT630- or the LAF237-treated mice when compared with that observed in tumors from vehicle control-treated mice (Supplemental Figure 3, B and C). Similar results were obtained using a TUNEL assay (data not shown). Importantly, PT630 had no direct effect on CT26 tumor cell viability in vitro as assessed by the 3-(4,5-dimethylthiazol-2-yl)-2,5-diphenyltetrazoliumbromide (MTT) assay (Supplemental Figure 3E), indicating that the decrease in tumor cell proliferation in vivo was due to an indirect effect on the transformed cells. We also assessed the effect of PT630 treatment on cell proliferation and apoptosis in the $\mathrm{K}$-ras ${ }^{\mathrm{G} 12 \mathrm{D}}$-driven lung tumors. PT630 reduced the percentage of Ki67-positive cells ( $P=0.006$; Figure 5, B and D) with no significant change in apoptosis (data not shown).
Based on our evidence that the antiproliferative effect of PT630 was indirect, we next compared the morphology of comparably sized $\left(40-50 \mathrm{~mm}^{2}\right)$ CT2 6 tumors from the 3 groups of mice. In tumors from vehicle control-treated mice, CT26 tumor cells appeared to be spindle shaped, and the tumors to be appeared highly organized (Figure 5A, bottom). Although tumors from mice treated with LAF237 appeared to be somewhat less organized than those from the saline-treated mice, the greatest impact on tumor morphology was observed in the tumors from PT630-treated mice, which were highly disorganized and less densely populated by tumor cells (Figure 5A, bottom).

Our morphologic data and prior evidence that FAP may have the potential to degrade collagen $(27,28)$, led us to hypothesize that deletion of FAP and inhibition of FAP activity may affect tumor growth by causing changes in the composition or organization of the ECM, leading to dysregulation of integrin-mediated signaling. Indeed, we found that tumors from Fap-null mice compared with those from Fap wild-type mice and tumors from mice treated with PT630 but not with LAF237 compared with those from vehicle 
A

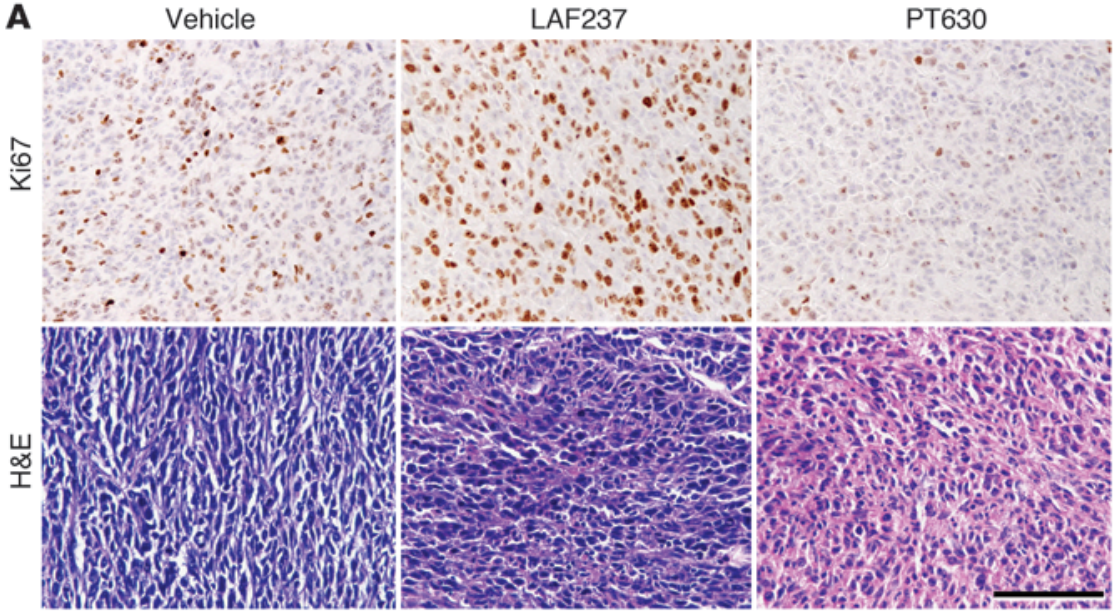

\section{Figure 5}

PT630 attenuates tumor cell proliferation and alters tumor morphology. (A and B) Sections of CT26 (A, top row) and endogenous lung tumors (B) isolated from mice, treated with either vehicle, LAF237, or PT630, were stained with Ki67 and H\&E. The morphology of CT26 tumors from the same groups of CT26 tumor-bearing mice was assessed based on H\&E staining (A, bottom row). Solid lines in B show representative tumor regions used to quantify cell proliferation in each group. Original magnification, $\times 40$. Scale bars: $100 \mu \mathrm{m}$. (C and D) Proliferative indices were calculated as percentage of Ki67-positive cells in CT26 (C; $n=10$ per group) and endogenous lung tumors (D; $n=5$ per group), respectively. Results are expressed as mean \pm SEM.
B

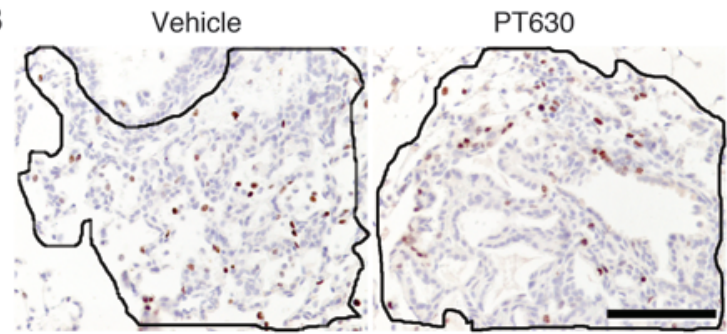

C

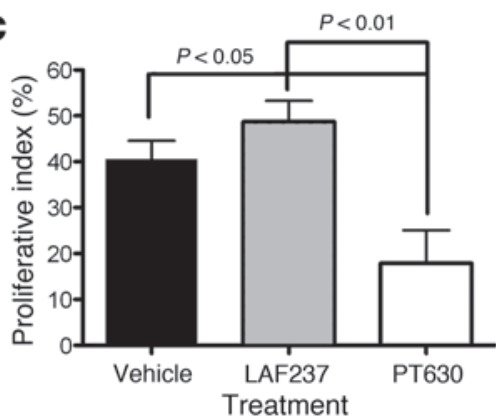

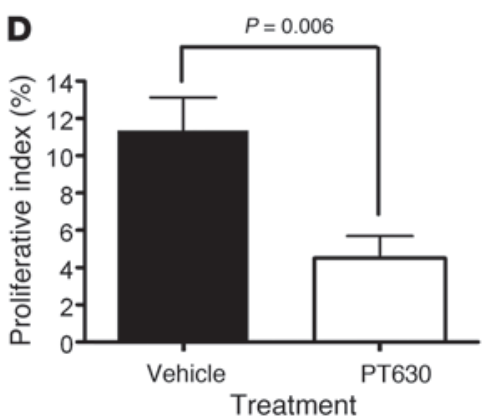

control-treated mice showed an increase in phospho-FAK ${ }^{\mathrm{Ty} r 397}$ and phospho-ERK (p44/42) (Figure 6 and Supplemental Figure 7). Although phosphorylation of MAPK usually correlates with cell proliferation, it can also increase the expression of the cell cycle inhibitor $\mathrm{p} 21^{\mathrm{WAF} 1}$ protein, causing cell cycle arrest $(44,45)$. We found that $\mathrm{p} 21^{\mathrm{WAF} 1}$ was indeed increased in FAP-null and PT630treated tumors $(P=0.04$; Figure 6 and Supplemental Figure 7). These data suggested that FAP may regulate cell proliferation, at least in part, via ECM/integrin-mediated signaling. These effects of FAP depletion and inhibition were fully recapitulated in the endogenous lung tumor model (Supplemental Figure 8).

FAP regulates tumor stromagenesis and angiogenesis. As deficiency in FAP expression or activity inhibited tumor cell growth indirectly and altered integrin-mediated signaling, we investigated its role in stromagenesis and, in particular, the content of myofibroblasts, which are an important source of ECM components and required for angiogenesis. We used immunohistochemistry to compare the density of myofibroblasts, which were identified using the conventional criteria of $\alpha$ Sma-expressing cells that were not in physical proximity to $\mathrm{CD} 34^{+}$endothelial cells
(46). We found that myofibroblasts were markedly less prevalent (5 fold) in tumors from PT630-treated mice than in tumors from control mice (Figure 7, A and B). We therefore conclude that FAP enzymatic activity regulates recruitment, proliferation, survival, or differentiation of myofibroblasts.

As angiogenesis, a key event in tumor progression, is dependent on ECM remodeling, proteases, and on the cell types that express FAP, fibroblasts, and pericytes $(47,48)$, we tested whether PT630 had an effect on CT26 tumor vascularization. Quantification of CD34+ vessels demonstrated that treatment with PT630 resulted in a 3-fold decrease in tumor vascularization compared with tumors from vehicle control- and LAF237-treated mice (Figure 7, A and B). These data indicate that FAP plays an important role in angiogenesis.

FAP activity regulates stromal collagen in vivo. The presence of collagen structures radially aligned with tumor cells has been suggested to promote tumor progression and invasion (49). Given the deficit in myofibroblasts we observed in tumors from mice treated with PT630 and, on the other hand, the reported in vitro collagenase activity of FAP, we investigated the net impact of inhibiting FAP on collagen organization and content in CT26 
A

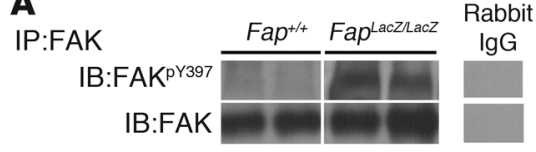

B

IP:ERK1/2

IB:phospho-ERK

IB:ERK $1 / 2$

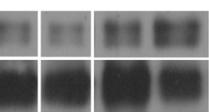

C

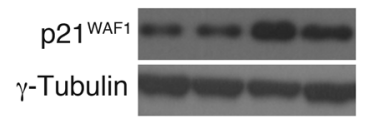

D

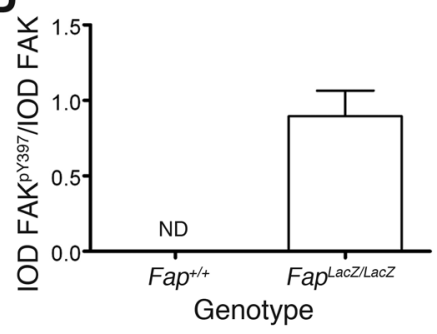

E
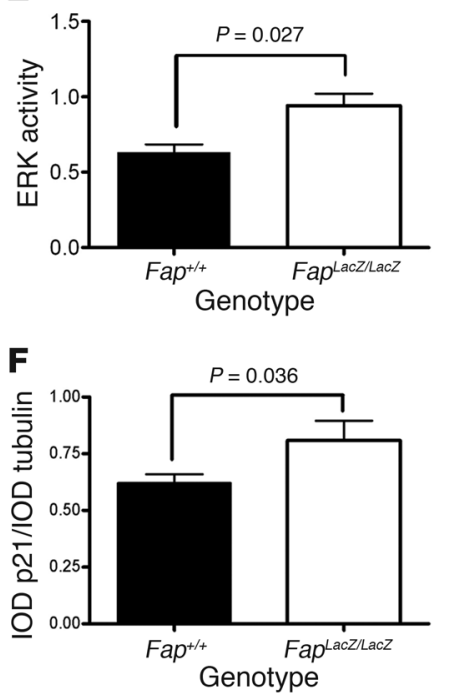

\section{Figure 6}

Deletion of FAP increases p21WAF1 via ECM-mediated signaling through FAK and ERK. FAK and ERK1/2 were immunoprecipitated from total extracts of CT26 tumors isolated from Fap ${ }^{+/+}$and Fap LacZ/LacZ mice, and the immune complexes were resolved by SDS-PAGE and immunoblotted for (A) phospho-FAK ${ }^{\mathrm{Y} 397}$ and total FAK and (B) ERK and phospho-ERK. (C) p21WAF1 immunoblot of total CT26 extracts from $\mathrm{Fap}^{+/+}$and FapLacZ/LacZ mice resolved by SDS-PAGE (2 representative samples from a total of 10 per group are shown for each immunoprecipitate/immunoblot). Lanes were run on the same gel but were noncontiguous (white lines). (D-F) Quantification by densitometry for all 10 samples from each group for each immunoprecipitate/ immunoblot. Results are expressed as mean \pm SEM. tumors. CT26 tumors harvested at similar size $\left(40-50 \mathrm{~mm}^{2}\right)$ from animals treated with saline, LAF237, or PT630 were stained with Picro-Sirius red, and collagen content of total tumor extracts was assessed by hydroxyproline assay. Picro-Sirius red, an elongated dye molecule, reacts with collagen and enhances its normal birefringence due to the fact that many dye molecules are aligned parallel to the long axis of each collagen molecule. The collagen fibers, in order of decreasing thickness corresponding to molecular disorganization, appear as red, orange, yellow, or green (50). When visualized under polarized light, collagenous structures with distinct birefringence were observed in both models. CT26 tumors in Fap ${ }^{L a c Z / L a c Z}$ mice exhibited dramatically increased birefringence (orange and yellow-green) and higher collagen content $\left(\sim 2\right.$ fold) than those from $\mathrm{Fap}^{+/+}$mice (Figure 8, A and B). Similarly, inhibition of FAP activity by treatment with PT630 resulted in an orange birefringence, suggesting a decrease in the organization of collagen when compared with the extent of birefringence observed in tumors from LAF237-treated mice and, even more so, when compared with the extent of birefringence observed in the tumors from vehicle control-treated mice (red birefringence; Supplemental Figure 9A). Furthermore, quantification of total collagen as determined by hydroxyproline content, demonstrated that collagen content was increased by $62 \%(P<0.01)$ in CT26 tumors from mice treated with PT630 (Supplemental Figure 9B), while LAF237 had no effect on collagen content when compared with tumors from mice treated with vehicle control.

\section{Figure 7}

PT630 inhibits angiogenesis and stromagenesis in CT26 tumors. (A) Sections of CT26 flank tumors were stained for CD34 (green), $\alpha$ Sma (red), and nuclei (DAPI; blue) and analyzed by epifluorescence microscopy. Original magnification, $\times 60$. Scale bar: $50 \mu \mathrm{m}$. (B) Blood vessels indicated by $\mathrm{CD}_{3} 4^{+}$endothelial cell and myofibroblasts $\left(\alpha \mathrm{Sma}{ }^{+}\right.$cells not in the proximity of CD34+ cells) were quantified from 10 tumors per group in 2 independent experiments. Results are expressed as mean \pm SEM.
Although tumor disorganization was not as obvious in H\&Estained lung tumors as it was in H\&E-stained CT26 tumors (Figure 1), the impact of FAP on collagen content in the lungs of endogenous tumor-bearing mice was comparable to that seen in the CT26 tumors, based on Picro-Sirius red birefringence microscopy and quantification of hydroxyproline content. $L S L-K$-ras ${ }^{G 12 D} ; \mathrm{Fap}^{+/+}$mice predominantly exhibited a red-orange birefringence, with some green birefringence, whereas tumors in $L S L-K-r^{G} s^{G 12 D} ; F a p^{L a c Z / L a c Z}$ mice exhibited a decrease in orange birefringence and a predominance of yellow-green birefringence (data not shown). Quantification of total collagen content revealed that although there was no difference in basal levels of collagen among the lungs of $L S L-K-\mathrm{ras}^{\mathrm{G} 12 D} ; \mathrm{Fap}^{+/+}, L S L-K-\mathrm{ras}^{\mathrm{G} 12 D} ; \mathrm{Fap}^{+/ L a c Z}$, and $L S L-K$-ras ${ }^{G 12 D} ; F a p^{L a c Z / L a c Z}$ mice (-Ad-Cre; Figure 8C), the levels of collagen that accumulated in tumor-bearing lungs from AdCre-infected $L S L-K$-ras ${ }^{G 12 D} ; F_{a} p^{+/ L a c Z}$ and $L S L-K$-ras ${ }^{G 12 D} ; F_{a p}{ }^{L a c Z / L a c Z}$ mice were significantly increased compared with Ad-Cre-infected
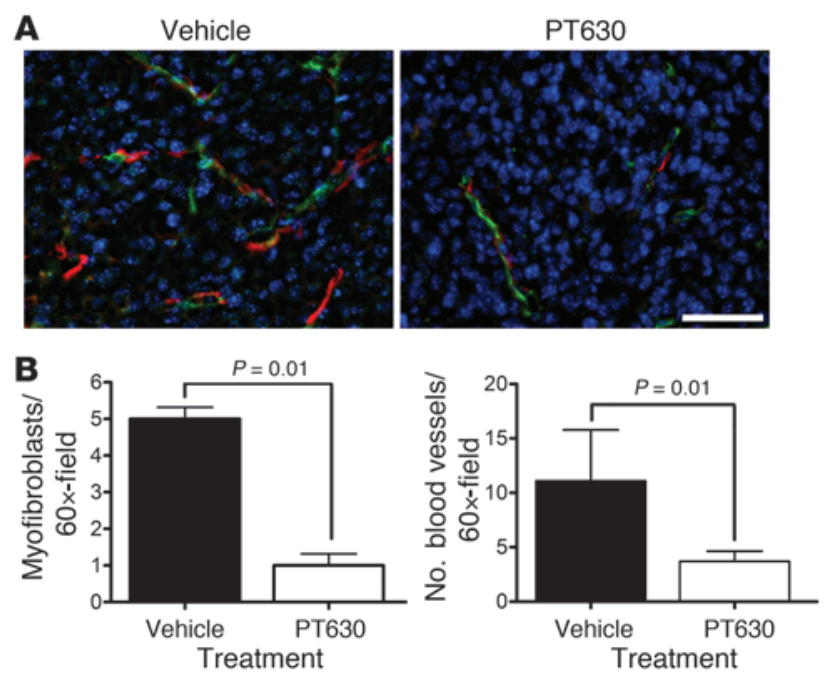
A
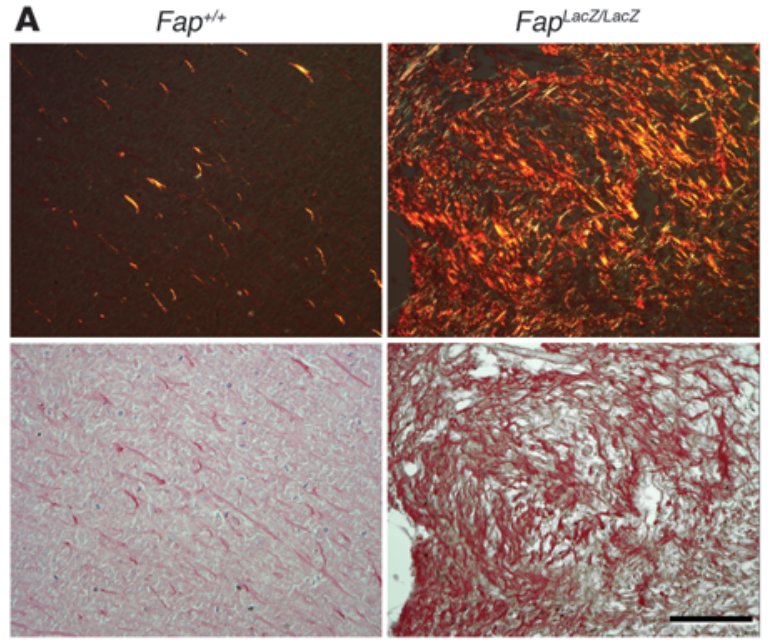

B

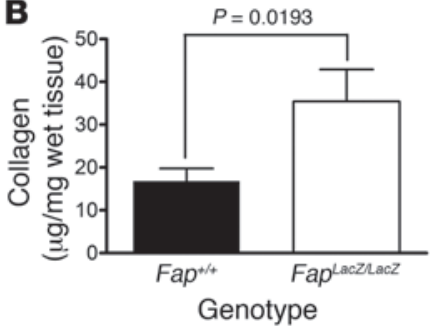

C

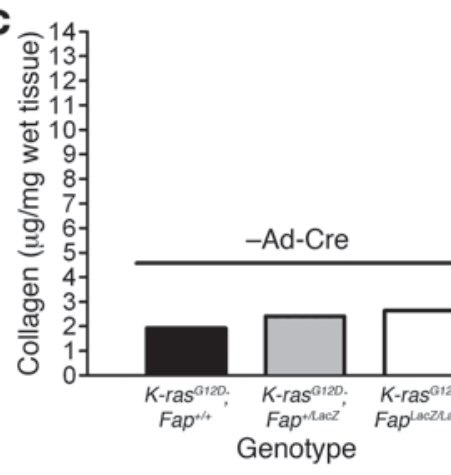

+Ad-Cre

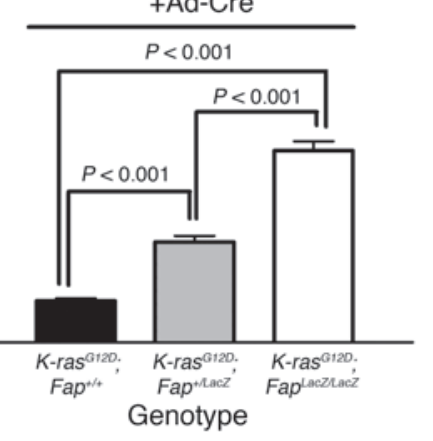

\section{Figure 8}

FAP regulates accumulation of collagen in vivo. (A) Sections of CT26 tumors were stained with Picro-Sirius red and visualized under polarized light (top row) and bright field (bottom row). The increase in orange birefringence in tumors from Fap LacZ/LacZ mice reflects less organized collagen. Original magnification, $\times 20$. Scale bar: $100 \mu \mathrm{m}$. (B) Collagen content of CT26 tumors $\left(100 \mathrm{~mm}^{2}\right)$ from Fap ${ }^{+/+}$and Fap ${ }^{\text {LacZ/LacZ }}$ mice. Data represent mean \pm SEM of 20 tumors per genotype. (C) Collagen content of lungs from uninfected $(n=3)$ mice and $L S L-K$-ras ${ }^{G 12 D} ; \mathrm{Fap}^{+/+}(n=6)$, LSL-K-ras G12D; Fap ${ }^{+/ L a c Z}(n=6)$, and LSL-K-ras ${ }^{G 12 D ; F a p^{L a c Z / L a c Z} \text { mice }(n=6)}$ 8 weeks after Ad-Cre infection. Results are expressed as mean \pm SEM.
$L S L-K-\operatorname{ras}^{\mathrm{G} 12 D} ; \mathrm{Fap}^{+/+}$mice $(P<0.001 ;$ Figure $8 \mathrm{C})$. A similar increase in collagen accumulation was observed in tumor-bearing $L S L-$ K-ras ${ }^{G 12 D} ; \mathrm{Fap}^{+/+}$mice treated with PT630 compared with those treated with vehicle control $(P<0.05$; Supplemental Figure 9C). Importantly, PT630 treatment had no effect on the collagen content of tumor-bearing lungs in $L S L-K$-ras ${ }^{G 12 D}$;Fap ${ }^{L a c Z / L a c Z}$ mice (Supplemental Figure 9C). Taken together, these data suggest that FAP endopeptidase activity enhances tumor growth, at least in part, by regulating stromal collagen content, leading to altered integrin-mediated signaling.

\section{Discussion}

Most epithelial-derived tumors are characterized by the generation of mesenchymal-derived stromal cells, including intratumoral and peritumoral TAFs and pericytes associated with the tumor vasculature. These stromal cells as well as the multiple products they release, such as TGF- $\beta$, ECM components, and proteases (particularly collagen and MMPs), have been implicated in tumor growth, angiogenesis, invasion, and metastasis. TAFs are phenotypically distinguishable from normal fibroblasts. Although their heterogeneity is yet to be fully explored, at least a subset of TAFs have been characterized as myofibroblasts based on expression of $\alpha \mathrm{Sma}$. FAP has also emerged as a marker of reactive fibroblasts in tumors as well as granulation tissue and in fibrotic lesions. Further studies are required to fully reveal the relationship between the SMA and FAP expressing populations in tumors, but consistent with other recent reports (51), our unpublished results suggest that they are overlapping but not coincident populations both in human and in mouse tumors. Recent studies have also established that TAFs are unique in their capacity to markedly enhance tumor growth compared with normal fibroblasts (52). These properties, taken together with the fact that the stromal cells are likely to be more genetically stable than tumor cells and the fact that they are common across multiple tumor types, suggest that therapies designed to target the protumorigenic mechanisms mediated by stromal cells, used in combination, may enhance the efficacy of tumor cell-targeted therapies. Thus, it is imperative that the mechanisms involved in stromal cell-dependent tumorigenic processes be delineated. In this study, we provide proof of principle that targeting a cell surface protease, FAP, expressed selectively on TAFs and pericytes inhibits tumor growth in diverse tumor types and that, at least in lung tumors, inhibition of the related dipeptidyl peptidase, DPPIV, also expressed on untransformed cells, can similarly inhibit tumor growth.

Recent interest in FAP stems from its tightly regulated expression, which is restricted to pericytes and fibroblasts induced to undergo differentiation within the microenvironment of tumors and in pathologic fibrosis (for example, in liver cirrhosis and idiopathic pulmonary fibrosis) $(20,21)$, and the potential of FAP to provide a common target in multiple tumor types. Two general approaches to targeting FAP have been taken in preclinical models and in patients. In antibody-based imaging studies, anti-FAP antibodies exhibited highly specific tumor targeting in patients (40). However, although a humanized anti-FAP antibody (mAb F19; sibrotuzumab) was well tolerated, it showed no efficacy in a phase II trial for metastatic colorectal cancer $(39,53)$. It is important to note, however, that this particular antibody does not inhibit FAP activity or have direct cytotoxic activity. On the other hand, it was 
recently reported that a monoclonal anti-FAP antibody conjugated to maytansinoid, FAP5-DM1, induced long-lasting inhibition of tumor growth and complete regressions in stroma-rich xenograft models of lung, pancreatic, and head and neck cancers in immune-deficient mice, with no evidence of toxicity (11). The latter data indicate that targeting FAP-expressing cells can effectively inhibit tumor growth but do not address the role of FAP itself in promoting tumor growth. Interestingly, Cheng and colleagues demonstrated that antibodies that inhibit the proteolytic activity of FAP-inhibited tumor growth in a xenograft model, although the mechanism involved was not determined (31).

The importance of the enzymatic activity has been addressed more directly using a second approach, employing inhibitors of FAP enzymatic activity. Administration of Val-boro-Pro (PT-100; Talabostat) attenuated tumor growth in a variety of tumor models in mice $(32,54)$. However, this particular compound inhibits multiple intracellular and extracellular dipeptidyl peptidases (e.g., FAP, CD26/DPPIV, DPP7), so that its effects could not be directly attributed to FAP. Finally, all the studies to date have been conducted in xenograft models of human tumors in immune incompetent mice, and in only one of these studies was the role of endogenous FAP expressed on stromal cells, rather than on tumors cells forced to overexpress FAP (31). In this study, we interrogated the role of endogenous FAP in vivo, by using Fap-null mice and by employing selective inhibitors of FAP and/or DPPIV. Collectively, our results establish that the enzymatic activity of FAP itself promotes tumor growth of both endogenous and syngeneic transplanted tumors in immune competent mice. Depletion of FAP expression and even partial inhibition of FAP enzymatic activity was sufficient to indirectly inhibit tumor cell proliferation. In addition, we demonstrate that FAP plays a critical role in tumor stromagenesis and angiogenesis. Furthermore, the excessive accumulation and disorganization of collagen we observed in FAP-deficient tumors indicates that FAP is an important source of collagenase activity and/or regulates other collagenases involved in modifying tumor-associated ECM, either directly or as the result of functioning in the recruitment or differentiation of critical collagenase-producing cells.

As an endogenous model, we chose to study lung tumors in $L S L-$ $K-$ ras $^{G 12 D}$ mice. The progression of the tumorigenic processes that develop in $L S L-K$-ras ${ }^{G 12 D}$ mice mimics that seen in human lung adenocarcinoma $(34,35,37)$. Among the different types of lung cancer in humans, adenocarcinoma is the most common type in the US and activating mutations of the $K$-ras oncogene are found in approximately one-third of human lung adenocarcinoma (55). This model also offers an advantage over spontaneous models in that tumor development in this model is controlled by the administration of Ad-Cre, which allowed administration of the pharmacologic inhibitors at a defined time after induction of expression of the oncogenic K-ras ${ }^{G 12 D}$ allele. Importantly, we found the development of hyperplasia and adenoma in this model was associated with the generation of tumor-associated myofibroblasts. Genetic deletion of FAP resulted in a marked reduction of K-ras ${ }^{\mathrm{G} 12 \mathrm{D}}$-driven tumors but had no impact on the phenotype of the tumor cells (Figures 1 and 2). Despite the obvious advantages of the $L S L-$ $K$-ras $^{G 12 D}$ model, there are also some limitations, including that, at least until mice expressing a conditional FAP allele are developed, the stage of tumorigenesis at which FAP is expressed cannot be controlled. Importantly, the protumorigenic role of FAP was also evident in CT26 colon tumors and Panc02 pancreatic tumors (data not shown) transplanted s.c. into immune competent syn- geneic hosts. Our results in these tumor models indicate that FAP is indeed an important factor in multiple tumor types and demonstrate that, even if FAP contributes to tumor initiation, it also plays a role in established tumors.

An important function of myofibroblasts is the deposition of ECM. Furthermore, studies have shown that collagen within tumors impacts tumor development $(56,57)$. More specifically, the presence of collagen structures radially aligned with tumor cells has been suggested to promote tumor progression and invasion (49). Given the deficit in myofibroblasts we observed in tumors from mice treated with PT630 and the important contribution of this cell type to ECM deposition, we expected we might find that tumors from these mice might also be depleted of ECM or that the organization of the ECM may be affected in these tumors. On the other hand, in view of the reported in vitro collagenase activity of FAP, we thought we might find that inhibiting FAP activity could lead to an accumulation of collagen. Interestingly, we found that the net impact of inhibiting or deleting FAP was to increase collagen accumulation.

The pharmacologic studies presented clearly establish that the enzymatic activity of FAP is critical to promotion of tumor growth by FAP in the K-ras-driven endogenous lung tumor model as well as in transplanted colon tumors. Furthermore, taken together with the results of the genetic approach, the inhibition of tumor growth by PT630 indicates that the inhibition of FAP enzymatic activity is sufficient to inhibit tumor growth. Interestingly, the results with the DPPIV-specific inhibitor, LAF237, indicate that this closely related member of the dipeptidyl peptidase family also promotes growth of endogenous lung tumors in $L S L-K$-ras ${ }^{G 12 D}$ mice, whereas inhibition of DPPIV alone had no effect on the s.c. transplanted colon tumor cells. It will be of interest in future studies to determine whether the contribution of DPPIV to tumorigenesis is restricted to certain tumor types or whether this disparity reflects differences in the requirements for the growth of endogenous and transplanted tumors.

Our data indicate that the inhibition of tumor growth in Fapnull and PT630-treated wild-type mice was due, at least in part, to reduction in tumor cell proliferation in both of the models that we studied. However, the fact that PT630 had no effect on the growth of cultured CT26 cells indicates that the effect in vivo was indirect, likely reflecting the impact we observed on the ECM, tumor stromagenesis, and/or angiogenesis, all of which are likely to be related.

Stromagenesis is a complex process that gives rise to an intricate network of TAFs, angiogenesis (both blood and lymphatic), and remodeling of the ECM. The TAFs in this "desmoplastic" stroma exhibit an altered phenotype, including higher proliferative index and, most notably, the enhanced production of collagens, hyaluronate, and epithelial growth factors (2). Induction of FAP expression is a prominent feature of TAFs in the vast majority of carcinomas $(13,16)$. FAP is also expressed on blood vessel-associated pericytes in tumors, and tumor vascularization is dependent on fibroblasts and ECM remodeling, while pericytes are required for vessel stabilization $(58,59)$. Interestingly, high levels of VEGF may negatively regulate pericytes and prevent vessel maturation. In this regard, it will be interesting to investigate whether FAP or DPPIV play a role in regulating VEGF, as dipeptidyl peptidases are known to activate other cytokines/growth factors (60). The data presented in this study establish that FAP plays an important role in tumor stromagenesis, vascularization, and ECM remodeling. The effect on stromagenesis may reflect a direct role for FAP in the recruit- 
ment, proliferation, survival, or differentiation of TAFs. These possibilities will be investigated in future experiments. Alternatively, FAP may indirectly regulate stromagenesis through effects on the tumor cells that induce stromagenesis or through modification of the ECM. Indeed, we found an accumulation of disorganized collagen fibrils in tumors from Fap-null mice and mice treated with PT630. TAFs and components of the matrix, including collagen, and more specifically, the presence of collagen structures radially aligned with tumor cells promote the growth of epithelial tumor cells and tumor progression and invasion $(1,49,56,57)$. Thus, the deficiency in TAFs and altered ECM evident in the tumors from Fap-null and PT630-treated mice likely contribute to the reduction in tumor growth. This is further evidenced by the increase we observed in the CDK inhibitor p21 levels in tumors from Fap-null and PT630-treated mice, as p21 is known to regulate the ECMmediated signaling pathway that activates cell cycle progression (44). The excess collagen accumulation, in the face of the reduction in SMA-expressing myofibroblasts in these tumors, may appear, at first glance, contradictory to the fact that myofibroblasts are believed to be a major source of collagen. But as discussed above, myofibroblasts may represent only a subset of TAFs and other TAFs may also be a major source of collagen. Furthermore, other cells, possibly the tumor cells themselves, may also contribute significantly to matrix deposition. However, as FAP has been shown to have collagenase activity in vitro, an alternative explanation for the excess accumulation of collagen may be that FAP is an important source or regulator of collagenase activity in tumors. In this case, the loss of FAP-dependent collagenase activity may prevent the expected cleavage and turnover of collagen produced by the residual TAFs. Another possibility is that FAP plays a role in the recruitment or differentiation of critical collagenase-producing cells. Finally, as FAP is not expressed on endothelial cells and vascularization is known to be dependent on fibroblasts and ECM remodeling $(47,52)$, it is likely that the reduction in TAFs and pericytes and/or the alterations in the ECM also underlie the reduced vascularization of tumors in FAP-null and PT630-treated mice.

In summary, this study provides proof of principle that targeting FAP can inhibit tumor growth in multiple tumor types, indirectly through effects on stromagenesis, vascularization, and ECM remodeling. Human epithelial-derived solid tumors are rich in FAP-expressing cells; for example, this is the case in colon, pancreatic, and also lung cancer, which is the leading cause of cancer-related deaths in both men and women worldwide $(12,15,34)$. Thus, in addition to establishing the mechanisms by which a specific stromal cell surface protease promotes tumorigenesis, these studies indicate that further exploring tumor stroma and, in particular, FAP as a potential therapeutic target in patients is warranted.

\section{Methods}

\section{Cell culture}

CT26.WT, a BALB/c-derived murine colon carcinoma cell line, was obtained from ATCC and grown as monolayer cultures in RPMI 1640 supplemented with $10 \%$ FCS, $10 \mathrm{mM}$ HEPES, $1 \mathrm{mM}$ sodium pyruvate, $2 \mathrm{mM}$ L-glutamine, $50 \mu \mathrm{g} / \mathrm{ml}$ gentamycin, and $20 \mu \mathrm{g} / \mathrm{ml}$ penicillin-streptomycin. Cells were maintained in a humid $5 \% \mathrm{CO}_{2}$ atmosphere at $37^{\circ} \mathrm{C}$.

\section{Animals}

Six- to eight-week-old female BALB/c and C57BL/6 mice were purchased from Charles River Laboratories. $L S L-K-$ ras $^{G 12 D}$ mice (34) were provided by
T. Jacks (Massachusetts Institute of Technology, Cambridge, Massachusetts, USA). FAP-deficient mice (Fap ${ }^{L a c Z / L a c Z}$; ref. 17) were obtained from W.J. Rettig and A. Schnapp (Boehringer Ingelheim Pharma KG, Ingelheim, Germany) and backcrossed 12 generations to $B A L B / c$. $L S L-K$-ras ${ }^{G 12 D}$ mice were crossed with FAP-deficient mice to generate Fap ${ }^{L a c Z / L a c Z}, F^{+} p^{+/ L a c Z}$, and littermate $\mathrm{Fap}^{+/+} L S L-K-$ ras $^{G 12 D}$ mice. Mice were genotyped as described in Supplemental Methods. All animal protocols used in this study were approved by The Wistar Institute's Institutional Animal Care and Use Committee, and all the procedures were conducted according to The Wistar Institute's ethical committee guidelines on animal welfare and the Guide for the care and use of laboratory animals (NIH publication no. 85-23. Revised 1985).

\section{Tumor models}

Endogenous lung tumor model. In the $L S L-K$-ras ${ }^{G 12 D}$ model, lung adenocarcinoma is initiated by Cre-mediated recombination via infection with an adenovirus expressing Cre recombinase (Ad-Cre; University of Iowa Gene Transfer Vector Core) by intranasal instillation as previously described $(33-35,37)$. Eight weeks after Ad-Cre infection, mice were sacrificed and lung tissue was harvested and analyzed histologically. For drug treatments, mice were randomized into 3 treatment groups, 4 weeks after infection. Saline solution (vehicle control), PT630 (75 $\mu \mathrm{g} / \mathrm{dose}$; patent application publication no. US 2007/0072830 A; PharmaForm LLC), or LAF237 (PharmaForm LLC) $(100 \mu \mathrm{g} /$ dose) was administered by oral gavage twice daily for an additional 4 weeks, at which time the lungs were harvested, processed, and analyzed histologically. The percentage of total lung area occupied by tumor in each of the 5 lobes was quantified by computer-assisted morphometry using Image-Pro 6.2 on H\&E-stained sections. For modified-"survival" studies depicted in the Kaplan-Meier plots, $L S L-K$-ras ${ }^{G 12 D} ; F_{a p}{ }^{+/+}(n=11), L S L-K-$ ras $^{G 12 D} ; F^{\prime} p^{+/ L a c Z}(n=17)$, and $L S L-$ $K-r a s^{G 12 D} ; F_{a p}{ }^{L a c Z / L a c Z}(n=16)$ mice were Ad-Cre infected and carefully monitored. Animals were sacrificed according to the guidelines set forth in The Wistar Institute guide for the use of animals (> $10 \%$ total body weight).

Syngeneic transplant tumor models. Six- to eight-week-old female FAP wildtype $\left(\mathrm{Fap}^{+/+}\right)$and FAP-null (Fap LacZ/LacZ) BALB/c mice (backcrossed 12 generations) were injected s.c. with $2 \times 10^{5} \mathrm{CT} 26$.WT cells, in the right and left flanks. For drug treatment, 2 days after tumor cell inoculation, mice were randomized into 3 treatment groups. Animals were treated by oral gavage $(200 \mu \mathrm{l})$ twice daily with saline solution (vehicle control), $100 \mu \mathrm{g} /$ dose PT630 (FAP and DPPIV inhibitor), or $100 \mu \mathrm{g} /$ dose LAF237 (Novartis DPPIV-specific inhibitor). Inhibitors were provided by Point Therapeutics Inc. Tumor growth was monitored every 2-3 days by measuring 2 perpendicular diameters using calipers. Experiments were performed with 7 mice per FAP genotype and in triplicates with 5-7 mice per drug treatment group.

\section{Ex vivo assay of FAP enzymatic activity}

FAP enzymatic activity in tumors was determined as previously described (32), using an immune-capture assay with Z-Gly-Pro-AMC (MP Biomedicals LLC) as a substrate. Ninety-six-well plates (Fluoronunc MaxiSorb) were coated overnight at $4^{\circ} \mathrm{C}$ with $200 \mu \mathrm{l}$ of $100 \mu \mathrm{g} / \mathrm{ml} \mathrm{rabbit} \mathrm{polyclonal}$ anti-FAP antibody (provided by J. Cheng, Fox Chase Cancer Center, Philadelphia, Pennsylvania, USA). Plates were then washed with PBS and blocked with 5\% BSA for 1 hour at room temperature. Tumors were excised and homogenized in MPER (Pierce Biotechnology Inc.) at $10 \mathrm{ml} / \mathrm{g}$ tumor. Samples were centrifuged at $18,204 \mathrm{~g}$ for 10 minutes. One milligram total protein extract of tumor was added to the coated wells (in a final volume of $200 \mu \mathrm{l}$ ), incubated for 1 hour, and washed with PBS containing $0.1 \%$ Tween-20. Reactions were carried out with $200 \mu \mathrm{M}$ Z-GlyProAMC (FAP, turnover number $/ K_{m}\left[k_{\text {cat }} / K_{m}\right], 5.3 \times 10^{4}-7.4 \times 10^{3} \mathrm{M}^{-1} \mathrm{~s}^{-1}$; DPPIV, $k_{\text {cat }} / K_{m}$, 9-15 $\left.\mathrm{M}^{-1} \mathrm{~s}^{-1}\right)(7,24)$ for 1 hour at room temperature. Fluorescence was measured (excitation and emission wavelengths of $355 \mathrm{~nm}$ and $460 \mathrm{~nm}$, 
respectively) using a Wallac 1420 Multilabel Counter (Perkin Elmer) and normalized to a milligram of tumor weight. Activity was expressed as relative fluorescence per milligram of tumor tissue per reaction time.

\section{Histology and immunohistochemical analyses}

CT26 flank tumors were harvested and paraffin embedded after fixation in Prefer (Anatech LTD) overnight at $4{ }^{\circ} \mathrm{C}$ followed by sucrose saturation. Lungs from $L S L-K-r a S^{G 12 D}$ mice were inflated intratracheally with $10 \%$ normal buffered formalin, fixed overnight, and embedded in paraffin. Four-micron sections were cut and stained with H\&E. For immunohistochemistry, paraffin-embedded sections were dewaxed, and antigen retrieval was performed in $10 \mathrm{mM}$ sodium citrate buffer, $\mathrm{pH} 7.4$, for 20 minutes at $95^{\circ} \mathrm{C}$. Slides were washed at room temperature and hydrated in PBS for 10 minutes. Endogenous peroxidase activity was then quenched with $3 \%$ hydrogen peroxidase for 10-20 minutes at room temperature. Sections were then blocked with $10 \%$ normal goat serum in PBS containing $1 \%$ BSA and $0.1 \%$ Tween- 20 for 1 hour. Endogenous avidin and biotin were blocked using the avidin-biotin kit (Vector Labs), following the manufacturer's instructions. Samples were then stained with antibodies against $\mathrm{Ki} 67(37 \mu \mathrm{g} / \mathrm{ml}$; M7249; DAKO), active caspase-3 (4 $\mu \mathrm{g} / \mathrm{ml}$; ab2302; Abcam), total ERK1/2 (1:25; 9102; Cell Signaling Technology), phosphoERK (1:200; 4370; Cell Signaling Technology), total

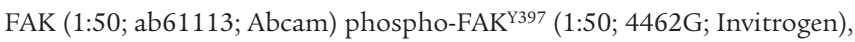

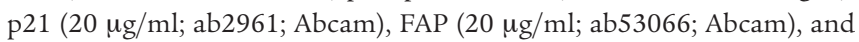
DPPIV (20 $\mu \mathrm{g} / \mathrm{ml}$; AF954; R\&D Systems), and isotype-matched antibodies were used as negative controls. Incubations were done overnight at $4^{\circ} \mathrm{C}$. Biotinylated secondary antibodies were used at $4 \mu \mathrm{g} / \mathrm{ml}$ and were detected with horseradish peroxidase, using the Vectastain Elite ABC (Vector Laboratories) as per manufacturer's instructions. The proliferative and apoptotic indices are expressed as the percentage of proliferative (Ki67-positive) and apoptotic (active caspase-3-positive) cells per total cells in the tumor sections. For CT26 tumors, the proliferative indices were based on 30 fields (objective magnification, $\times 40$ ), including 10 fields from each of 3 distinct regions per tumor. Proliferation indices of lung tumors were calculated based on 10 tumor nodules in each mouse ( 2 from each of 5 lobes), identified by morphology.

\section{Immunofluorescence}

Paraffin-embedded sections $(4 \mu \mathrm{m})$ were dewaxed, followed by antigen retrieval in $10 \mathrm{mM}$ sodium citrate buffer for 30 minutes at $95^{\circ} \mathrm{C}$, with sections covered with $10 \mathrm{mM}$ sodium citrate buffer. Clara cells and alveolar type II cells were stained as described previously (38), using the polyclonal antibody anti-CC10 (4 $\mu \mathrm{g} / \mathrm{ml}$; sc9772; Santa Cruz Biotechnology Inc.) and the polyclonal rabbit antibody anti-proSPC (1:200; AB3786; Chemicon), respectively. CT26 paraffin-embedded tumor sections were dewaxed, followed by antigen retrieval as described above. To block mouse IgG, the M.O.M. kit (Vector Laboratories) was employed. The antibody against mouse Sma (0.4 $\mu \mathrm{g} / \mathrm{ml}$; clone 1A4; M0851; DAKO) in M.O.M. diluent and incubated for 1 hour at $37^{\circ} \mathrm{C}$. After 3 washes with phosphate buffer at pH 7.2 containing Tween-20 ( $0.1 \%$ ) (5 minutes), biotinylated mouse IgG was added and incubated for 30 minutes at $37^{\circ} \mathrm{C}$. Following 3 washes, texas red-streptavidin (Vector Laboratories) was incubated for 1 hour at $37^{\circ} \mathrm{C}$. After washing, sections were incubated with CD34 (4 $\mu \mathrm{g} / \mathrm{ml}$; clone MEC14.7; Abcam) overnight at $4^{\circ} \mathrm{C}$. Secondary antibody (Alexa Fluor 488; Invitrogen) was applied to sections for 1 hour at $37^{\circ} \mathrm{C}$. Nuclei were counterstained with DAPI for 5 minutes. $\mathrm{SMA}^{+}$myofibroblasts and pericytes and $\mathrm{CD} 34^{+}$blood vessels were quantified (magnification, $\times 60$ ) for 10 animals per group from 2 independent experiments as described above.

\section{Immunoprecipitation and immunoblotting}

CT26 tumors were dissected and lysed by homogenization in $25 \mathrm{mM}$ HEPES, pH 7.5, containing $0.1 \%$ Triton X-100, $300 \mathrm{mM} \mathrm{NaCl}, 0.5 \mathrm{mM}$
DTT, $0.2 \mathrm{mM}$ EDTA, $1.5 \mathrm{mM} \mathrm{MgCl}_{2}$, containing $20 \mathrm{mM} \beta$-glycerophosphate, $1 \mathrm{mM} \mathrm{Na}_{3} \mathrm{VO}_{4}, 10 \mathrm{mM} \mathrm{NaF}, 10 \mathrm{mM}$ sodium pyrophosphate, and protease inhibitor cocktail (Roche) at $25 \mathrm{mg} / \mathrm{ml}$. After incubation on ice for 20 minutes, samples were centrifuged at 13,000 g. Equivalent amounts $(25 \mu \mathrm{g})$ of protein (assayed with BCA protein assay; Pierce Biotechnology Inc.) were resolved by SDS-PAGE, transferred to PVDF membranes (Millipore), and probed with indicated antibodies. Incubation with primary antibodies (anti-p21 clone C19 or anti-FAP; Santa Cruz Biotechnology Inc. and J. Cheng, respectively) and secondary antibodies in $5 \%$ milk in phosphate buffer containing $0.1 \%$ Tween-20, with washes in phosphate buffer containing $0.1 \%$ Tween- 20 between steps. For immunoprecipitation, equal amounts of protein $(1 \mathrm{mg})$ were precleared using anti-rabbit antibody for 90 minutes at $4{ }^{\circ} \mathrm{C}$, followed by incubation with protein A-agarose (Pierce Biotechnology Inc.). Precleared lysates were subjected to immunoprecipitation at $4{ }^{\circ} \mathrm{C}$ overnight with indicated antibodies. Immune complexes were recovered using protein A-agarose. Products were fractionated as above and blots were probed with anti-phosphoFAK ${ }^{\mathrm{PY} 397}$ or anti-phosphoERK antibody. Primary and secondary antibodies were diluted in blocking buffer (4\% BSA in $30 \mathrm{mM}$ Tris- $\mathrm{HCl}, \mathrm{pH} 7.6,75 \mathrm{mM} \mathrm{NaCl}, 0.05 \%$ Tween-20), with washes in $30 \mathrm{mM}$ Tris- $\mathrm{HCl}, \mathrm{pH} 7.6,75 \mathrm{mM} \mathrm{NaCl}, 0.05 \%$ Tween-20. Blots were washed and incubated in stripping buffer (2\% SDS, $62.5 \mathrm{mM}$ Tris, pH 6.8, $100 \mathrm{mM}$ $\beta$-mercaptoethanol) at $50^{\circ} \mathrm{C}$ for 30 minutes or run in parallel and reprobed with primary antibodies against FAK or $\mathrm{ERK}_{1 / 2}$. Detection was conducted using ECL (GE Healthcare). Protein levels (expressed as the image optical density) were determined by densitometry using ImagePro. ERK activity and active FAK were expressed as ratio of phosphorylated form relative to the total fraction.

\section{Analyses of collagen content of tumors}

Picro-Sirius red staining. Serial sections of CT26 tumors were dewaxed as described above. Slides were incubated in picric acid-saturated solution containing $0.01 \%$ Sirius red (Sigma-Aldrich) for 1 hour in a humidified chamber at room temperature. After washing with $1 \%$ acetic acid (15 minutes) and water (1 minute), slides were alcohol dehydrated, mounted, and visualized on a Leica Microscope under 90-degree polarized light.

Hydroxyproline assay. CT26 tumors $(50 \mathrm{mg})$ were cut and digested overnight at $110^{\circ} \mathrm{C}$ in $1 \mathrm{ml}$ of $6 \mathrm{~N} \mathrm{HCl}$. After neutralization with $6 \mathrm{~N} \mathrm{NaCl}$, the $\mathrm{pH}$ was adjusted $(6.0<\mathrm{pH}<10.0)$. Samples $(100 \mu \mathrm{l})$ were mixed with $1 \mathrm{ml}$ chloramine $\mathrm{T}$ solution ( $1.4 \%$ chloramine $\mathrm{T}, 10 \%$ isopropanol, $0.5 \mathrm{M}$ sodium acetate, $\mathrm{pH}$ 6.0) for 20 minutes at room temperature, followed by $1 \mathrm{ml}$ Erlich's solution (14.9\% p-dimethylaminobenzaldehyde, $70 \%$ isopropanol, $20 \%$ perchloric acid; Sigma-Aldrich) and incubation at $65^{\circ} \mathrm{C}$ for $15 \mathrm{~min}$ utes. Aliquots $(200 \mu \mathrm{l})$ were transferred to 96 -well plates, and absorbance was measured at $570 \mathrm{~nm}$. Collagen content was calculated by comparison with a standard curve generated with cis-4 hydroxy-L-proline $(0.01-110 \mu \mathrm{g} /$ $\mathrm{ml}$; Sigma-Aldrich), using the conversion factor of $1 \mu \mathrm{g}$ hydroxyproline, corresponding to $6.94 \mu \mathrm{g}$ collagen (61). Total collagen was expressed as $\mu \mathrm{g}$ collagen $\mathrm{mg}^{-1}$ wet tissue.

\section{Statistics}

All results are expressed as mean \pm SEM. Statistical analysis was performed using 1-way ANOVA with the Tukey's multiple comparison test and 2 -tailed Student's $t$ test (GraphPad Prism). $P$ values of less than 0.05 were considered statistically significant.

\section{Acknowledgments}

Inhibitors (PT630 and LAF237 [PharmForm LLC]) were provided by Point Therapeutics Inc. The authors acknowledge W. Lee (University of Pennsylvania) for his technical assistance on the vascu- 
larization analysis. We also thank F.S. Keeney and J. Hayden (The Wistar Institute Microscopy Core) for their assistance with the imaging data; R. Delgiacco (The Wistar Institute Histotechnology Core) for the processing of tissues; Denise DiFrancesco, Director of The Wistar Animal Facility; and S. Berliner for help in preparing the manuscript. This work was supported by NIH grant T32 CA09171, a Wistar Cancer Training Grant, a grant from the Pennsylvania Department of Health, and Point Therapeutics Inc. A.M. Santos was supported by the Irvington Institute Fellowship Program of the Cancer Research Institute.
Received for publication February 20, 2009, and accepted in revised form September 28, 2009.

Address correspondence to: Ellen Puré or Angélica M. Santos, The Wistar Institute, 3601 Spruce Street, Philadelphia, Pennsylvania 19104, USA. Phone: (215) 898-1570; Fax: (215) 898-3937; E-mail: pure@wistar.org (E. Puré); asantos@wistar.org (A.M. Santos).

Nazneen Aziz's present address is: Vitrimark, Providence, Rhode Island, USA.
1. Bhowmick, N.A., Neilson, E.G., and Moses, H.L. 2004. Stromal fibroblasts in cancer initiation and progression. Nature. 432:332-337.

2. Tlsty, T.D., and Coussens, L.M. 2006. Tumor stroma and regulation of cancer development. Annu. Rev. Pathol. 1:119-150.

3. Lopez-Otin, C., and Matrisian, L.M. 2007. Emerging roles of proteases in tumour suppression. Nat. Rev. Cancer. 7:800-808.

4. Blouse, G.E., et al. 2009. A novel mode of intervention with serine protease activity: Targeting zymogen activation. J. Biol. Chem. 284:4647-4657.

5. Wallrapp, C., et al. 2000. A novel transmembrane serine protease (TMPRSS3) overexpressed in pancreatic cancer. Cancer Res. 60:2602-2606.

6. DeClerck, Y.A., and Imren, S. 1994. Protease inhibitors: role and potential therapeutic use in human cancer. Eur. J. Cancer. 30A:2170-2180.

7. Edosada, C.Y., et al. 2006. Peptide substrate profiling defines fibroblast activation protein as an endopeptidase of strict Gly2-Pro1-cleaving specificity. FEBS Lett. 580:1581-1586.

8. Edosada, C.Y., et al. 2006. Selective inhibition of fibroblast activation protein protease based on dipeptide substrate specificity. J. Biol. Chem. 281:7437-7444.

9. Hu, Y., et al. 2005. Synthesis and structure-activity relationship of N-alkyl Gly-boro-Pro inhibitors of DPP4, FAP, and DPP7. Bioorg. Med. Chem. Lett. 15:4239-4242.

10. Meadows, S.A., et al. 2007. Ala657 and conserved active site residues promote fibroblast activation protein endopeptidase activity via distinct mechanisms of transition state stabilization. Biochemistry. 46:4598-4605.

11. Ostermann, E., et al. 2008. Effective immunoconjugate therapy in cancer models targeting a serine protease of tumor fibroblasts. Clin. Cancer Res. 14:4584-4592.

12. Cohen, S.J., et al. 2008. Fibroblast activation protein and its relationship to clinical outcome in pancreatic adenocarcinoma. Pancreas. 37:154-158.

13. Garin-Chesa, P., Old, L.J., and Rettig, W.J. 1990. Cell surface glycoprotein of reactive stromal fibroblasts as a potential antibody target in human epithelial cancers. Proc. Natl. Acad. Sci.U. S. A. 87:7235-7239.

14. Goscinsky, M.A., et al. 2008. FAP-alpha and uPA show different expression patterns in premalignant and malignant esophageal lesions. Ultrastruct. Pathol. 32:89-96.

15. Henry, L.R., et al. 2007. Clinical implications of fibroblast activation protein in patients with colon cancer. Clin. Cancer Res. 13:1736-1741.

16. Scanlan, M.J., et al. 1994. Molecular cloning of fibroblast activation protein a, a member of the serine protease family selectively expressed in stromal fibroblasts of epithelial cancers. Proc. Natl. Acad. Sci. U. S. A. 91:5657-5661.

17. Niedermeyer, J., et al. 2000. Targeted disruption of mouse fibroblast activation protein. Mol. Cell. Biol. 20:1089-1094

18. Mathew, S., et al. 1995. The gene for fibroblast activation protein [alpha] (FAP), a putative cell surfacebound serine protease expressed in cancer stroma and wound healing, maps to chromosome band 2q23. Genomics. 25:335-337.

19. Levy, M.T., et al. 1999. Fibroblast activation protein: a cell surface dipeptidyl peptidase and gelatinase expressed by stellate cells at the tissue remodelling interface in human cirrhosis. Hepatology. 29:1768-1778.

20. Wang, X.M., et al. 2008. Fibroblast activation protein and chronic liver disease. Front. Biosci. 13:3168-3180.

21. Acharya, P.S., Zukas, A., Chandan, V., Katzenstein, A.L., and Puré, E. 2006. Fibroblast activation protein: a serine protease expressed at the remodeling interface in idiopathic pulmonary fibrosis. Hum. Pathol. 37:352-360.

22. Rettig, W.J., et al. 1993. Regulation and heteromeric structure of the fibroblast activation protein in normal and transformed cells of mesenchymal and neuroectodermal origin. Cancer Res. 53:3327-3335.

23. Huber, M.A., et al. 2003. Fibroblast activation protein: differential expression and serine protease activity in reactive stromal fibroblasts of melanocytic skin tumors. J. Invest. Dermatol. 120:182-188.

24. Aertgeerts, K., et al. 2005. Structural and kinetic analysis of the substrate specificity of human fibroblast activation protein alpha. J. Biol. Chem. 280:19441-19444.

25. Havre, P.A., et al. 2008. The role of CD26/dipeptidyl peptidase IV in cancer. Front. Biosci. 13:1634-1645.

26. Rettig, W.J., et al. 1994. Fibroblast activation protein: purification, epitope mapping and induction by growth factors. Int. J. Cancer. 58:385-392.

27. Christiansen, V.J., Jackson, K.W., Lee, K.N., and McKee, P.A. 2007. Effect of fibroblast activation protein and [alpha]2-antiplasmin cleaving enzyme on collagen types I, III, and IV. Arch. Biochem. Biophys. 457:177-186.

28. Aggarwal, S., et al. 2008. Fibroblast activation protein peptide substrates identified from human collagen I derived gelatin cleavage sites. Biochemistry. 47:1076-1086.

29. Piñeiro-Sánchez, M.L., et al. 1997. Identification of the $170-\mathrm{kDa}$ melanoma membrane-bound gelatinase (seprase) as a serine integral membrane protease. J. Biol. Chem. 272:7595-7601.

30. Park, J.E., et al. 1999. Fibroblast activation protein, a dual specificity serine protease expressed in reactive human tumor stromal fibroblasts. J. Biol. Chem. 274:36505-36512.

31. Cheng, J.D., et al. 2002. Promotion of tumor growth by murine fibroblast activation protein, a serine protease, in an animal model. Cancer Res. 62:4767-4772.

32. Cheng, J.D., et al. 2005. Abrogation of fibroblast activation protein enzymatic activity attenuates tumor growth. Mol. Cancer Ther. 4:351-360.

33. Grimm, J., et al. 2005. Use of gene expression profiling to direct in vivo molecular imaging of lung cancer. Proc. Natl. Acad. Sci. U. S. A. 102:14404-14409.

34. Jackson, E.L., et al. 2001. Analysis of lung tumor initiation and progression using conditional expression of oncogenic K-ras. Genes Dev. 15:3243-3248.

35. Johnson, L., et al. 2001. Somatic activation of the $\mathrm{K}$-ras oncogene causes early onset lung cancer in mice. Nature. 410:1111-1116.

36. Kissil, J.L., et al. 2007. Requirement for Rac1 in a K-ras-induced lung cancer in the mouse. Cancer Res. 67:8089-8094.

37. Tuveson, D.A., et al. 2004. Endogenous oncogenic K-ras (G12D) stimulates proliferation and widespread neoplastic and developmental defects. Cancer Cell. 5:375-387.

38. Besson, A., et al. 2007. Discovery of an oncogenic activity in p27Kip1 that causes stem cell expansion and a multiple tumor phenotype. Genes Dev. 21:1731-1746.

39. Scott, A.M., et al. 2003. A phase I dose-escalation study of sibrotuzumab in patients with advanced or metastatic fibroblast activation protein-positive cancer. Clin.Cancer Res. 9:1639-1647.

40. Welt, S., et al. 1994. Antibody targeting in metastatic colon cancer: a phase I study of monoclonal antibody F19 against a cell-surface protein of reactive tumor stromal fibroblasts. J. Clin. Oncol. 12:1193-1203.

41. Narra, K., et al. 2006. Inhibitors of the stromal protease fibroblast activation protein attenuate tumor growth in vivo [abstract]. Proc. Amer. Assoc. Cancer Res. 47:4382.

42. Brandt, I., et al. 2005. Inhibition of dipeptidylpeptidase IV catalyzed peptide truncation by Vildagliptin ((2S)-\{[(3-hydroxyadamantan-1yl)amino]acetyl\}-pyrrolidine-2-carbonitrile). Biochem. Pharmacol. 70:134-143.

43. Villhauer, E.B., et al. 2003. 1-[[(3-hydroxy-1adamantyl)amino]acetyl]-2-cyano-(S)-pyrrolidine: a potent, selective, and orally bioavailable dipeptidyl peptidase IV inhibitor with antihyperglycemic properties. J. Med. Chem. 46:2774-2789.

44. Bryant, P., Zheng, Q., and Pumiglia, K. 2006. Focal adhesion kinase controls cellular levels of p27/Kip1 and p21/Cip1 through Skp2-dependent and -independent mechanisms. Mol. Cell. Biol. 26:4201-4213.

45. Pumiglia, K.M., and Decker, S.J. 1997. Cell cycle arrest mediated by the MEK/mitogen-activated protein kinase pathway. Proc. Natl. Acad. Sci. U. S. A. 94:448-452.

46. Eyden, B., Banerjee, S.S., Shenjere, P., and Fisher, C. 2009. The myofibroblast and its tumours. J. Clin. Pathol. 62:236-249.

47. Gerhardt, H., and Betsholtz, C. 2003. Endothelialpericyte interactions in angiogenesis. Cell Tissue Res. 314:15-23.

48. Noma, K., et al. 2008. The essential role of fibroblasts in esophageal squamous cell carcinoma-induced angiogenesis. Gastroenterology. 134:1981-1993.

49. Provenzano, P.P., et al. 2006. Collagen reorganization at the tumor-stromal interface facilitates local invasion. BMC Med. 4:38.

50. Rich, L., and Whittaker, P. 2005. Collagen and picrosirius red staining: a polarized light assessment of fibrillar hue and spatial distribution. Braz. J. Morphol. Sci. 22:97-104.

51. Kalluri, R., and Zeisberg, M. 2006. Fibroblasts in cancer. Nat. Rev. Cancer. 6:392-401.

52. Orimo, A., et al. 2005. Stromal fibroblasts present in invasive human breast carcinomas promote tumor growth and angiogenesis through elevated 
SDF-1/CXCL12 secretion. Cell. 121:335-348.

53. Hofheinz, R.D., et al. 2003. Stromal antigen targeting by a humanised monoclonal antibody: an early phase II trial of sibrotuzumab in patients with metastatic colorectal cancer. Onkologie. 26:44-48.

54. Adams, S., et al. 2004. PT-100, a small molecule dipeptidyl peptidase inhibitor, has potent antitumor effects and augments antibody-mediated cytotoxicity via a novel immune mechanism. Cancer Res. 64:5471-5480.

55. Ding, L., et al. 2008. Somatic mutations affect key pathways in lung adenocarcinoma. Nature. 455:1069-1075.

56. Armstrong, T., et al. 2004. Type I collagen promotes the malignant phenotype of pancreatic ductal adenocarcinoma. Clin. Cancer Res. 10:7427-7437.

57. Moro, L., Arbini, A.A., Marra, E., and Greco, M. 2005. Down-regulation of BRCA2 expression by collagen type I promotes prostate cancer cell proliferation. J. Biol. Chem. 280:22482-22491.

58. Willett, C.G. 2004. Direct evidence that the VEGF-specific antibody bevacizumab has antivascular effects in human rectal cancer. Nat. Med. 10:145-147.

59. Morikawa, S., et al. 2002. Abnormalities in pericytes on blood vessels and endothelial sprouts in tumors. Am. J. Pathol. 160:985-1000.

60. Bogenrieder, T., and Herlyn, M. 2002. Cell-surface proteolysis, growth factor activation and intercellular communication in the progression of melanoma. Crit. Rev. Oncol. Hematol. 44:1-15.

61. Jackson, D.S., and Cleary, E.G. 1967. The determination of collagen and elastin. Methods Biochem. Anal. 15:25-76. 